\title{
Cod liver oil/ honey mixture: An effective treatment of equine complicated lower leg wounds
}

\author{
Magda mahmoud Ali* ${ }^{1}$ and Khaled Radad ${ }^{2}$ \\ 1. Department of Surgery, 2. Department of Pathology, \\ Faculty of Veterinary Medicine, Assiut University, Assiut 71526, Egypt \\ * Corresponding author email : magdaali70@yahoo.com \\ Published online : 30-04-2011
}

\begin{abstract}
Wounds are of great concern in animals as they affect animal productivity and their treatment represents an economic burden to the owners particularly in developing countries. The present study investigated the beneficial role of a mixture of cod liver oil and honey in treatment of old wounds in equines. In which, 3 horses and 7 donkeys were admitted to the Veterinary Teaching Hospital at Assiut University, Assiut, Egypt with old wounds at the metacarpus (5 cases) and metatarsus ( 3 cases) and at the level of fetlock and carpal joints (one case each). Treatment of these wounds with cod liver oil/honey mixture markedly decreased their sizes and resulted in formation of a healthy scar after one month of treatment. To explain the mechanisms that underlie the effects of this mixture, 9 surgically-induced wounds at the metacarpus in 9 donkeys were treated with honey, cod liver oil or the mixture ( 3 wounds each) and compared with parallel untreated control wounds at the metatarsus. It was found that treatment of wounds with honey, cod liver oil and the mixture apparently decreased the size of wounds. Histopathological examination revealed that treated wounds with honey or cod liver oil showed complete epithelization over the wound areas. On the other hand, epithelization process was not complete in wounds treated with the mixture. Granulation tissue showed some variability between different treatments. Of which, wounds treated with the mixture showed a higher degree of maturity with increasing numbers of fibrocytes and parallel collagenous fibers. This could underlie the good results obtained in case studied wounds with the mixture. In conclusion, mixture of cod liver oil and honey is beneficial in treatment of old traumatized wounds in equine and this effect is primarily mediated by formation of healthy and mature scars. Cod liver oil or honey alone is beneficial for healing of recent and surgical wounds as they facilitate epithelization process.
\end{abstract}

Keywords: Equine, Wound, Healing, Honey, Cod liver oil.

\section{I ntroduction}

Repair of wound is complex series of regulated molecular and cellular events that begin early at the time of injury and end with complete restoring of the structural integrity of damaged tissue. Healing process usually depends on the kind, location and cleanness of the wound, presence of infection and time of treatment. Moreover, the way of wound management affects the rate of healing, the final cosmetic appearance and the satisfaction of customers (Liptak, 1997).

In equines, wounds on both metacarpus and metatarsus are common and often left to heal by second intention because of excessive skin tension and contamination (Bigbie et.al., 1991). Also, these wounds form an excessive granulation tissue and heal after prolonged time because they have a longer preparatory phase of healing, greater retraction of the margins and slower rates of wound contraction and epithelization compared with other wounds in the body (Jacobs et al., 1984).

Many therapeutic agents are used for topical treatment of wounds including yeast cell derivatives (Crowe et al., 1999), cod liver oil (Kietzmann and Braun, 2006), honey (Iftikar et al., 2009), sugar (Cavazana et al., 2009), corticosteroids (Jorissen and Bachert, 2009) and phenytoin (Qunaibi et al., 2009). Of which, honey and cod liver oil are increasingly used as natural products and biological therapies in clinical practice. However, bee honey has been used since ancient times, Egyptian times circa $2000 \mathrm{BC}$, to accelerate wound healing, modern honey wound dressings have become more widely available and used in wound management (Zumla and Lulat, 1989). This is largely due to the growing clinical problems of antibiotic-resistant bacteria and the combined difficulties for the practitioners in managing chronic wounds such as burns and leg ulcers (Lay-flurrie, 2008). Besides antimicrobial effects of honey (Cooper and Molan, 1999), it has anti-inflammatory and antioxidant properties (Gheldof and Engeseth, 2002), promotes moist wound healing and facilitates debridement (Majtán, 2009; Pieper, 2009). Cod liver 
Cod liver oil/honey mixture: An effective treatment of equine complicated lower leg wounds

Table-1. Mean size of case study wounds ( 3 horses and 7 donkeys) before and after treatment with mixture of cod liver oil and honey.

\begin{tabular}{lll}
\hline Animals & Before treatment & After treatment \\
\hline Horses & $8.0 \pm 6.8=54.40 \mathrm{~cm}^{2}$ & $3.8 \pm 2.8=10.64 \mathrm{~cm}^{2}$ \\
Donkeys & $7.1+6.3=44.73 \mathrm{~cm}^{2}$ & $3.9+3.2=12.48 \mathrm{~cm}^{2}$ \\
\hline
\end{tabular}

Table-2. Mean size of surgically-induced wounds in donkeys as the result of treatment with honey, cod liver oil or the mixture ( 3 metacarpal wounds each) compared to control wounds ( 3 metatarsal wounds).

\begin{tabular}{lcccc}
\hline Substances & \multicolumn{2}{c}{ After two weeks } & \multicolumn{2}{c}{ After one month } \\
\cline { 2 - 4 } & Treated wounds & Control wounds & Treated wounds & Control wounds \\
\hline Honey & $9.50 \mathrm{~cm}^{2}$ & $10.99 \mathrm{~cm}^{2}$ & $6.59 \mathrm{~cm}^{2}$ & $8.79 \mathrm{~cm}^{2}$ \\
Cod liver oil & $9.42 \mathrm{~cm}^{2}$ & $10.68 \mathrm{~cm}^{2}$ & $6.60 \mathrm{~cm}^{2}$ & $8.79 \mathrm{~cm}^{2}$ \\
Mixture & $9.90 \mathrm{~cm}^{2}$ & $10.99 \mathrm{~cm}^{2}$ & $6.20 \mathrm{~cm}^{2}$ & $8.16 \mathrm{~cm}^{2}$ \\
\hline
\end{tabular}

oil is a nutritional supplement derived from liver of cod fish. It has high levels of omega 3 fatty acid, vitamin A and vitamin D. Terkelsen et al. (2000) reported that cod liver oil was beneficial in wound healing as it enhances epithelization and neovascularization.

This study was built up to investigated the beneficial effects of the mixture of cod liver oil and honey in healing of old, heavily traumatized and contaminated wounds in equines and the histopathological bases for such effects.

\section{Materials and methods}

All experimental procedures in this study were approved by the Ethical Committee on Animal Experimentation in Assiut University, Assiut 71526, Egypt.

Treatment of complicated wounds in admitted animals with mixture of cod liver oil and honey History of cases: Ten animals ( 3 horses and 7 donkeys) were presented to the Veterinary Teaching Hospital, Faculty of Veterinary Medicine, Assiut University, Assiut, Egypt early in 2010. Upon examination, all animals were suffering of old and heavily traumatized wound with exuberant granulation tissues and infection of the wound surface. Wounds were located on metacarpus ( 3 horses and 2 donkeys), metatarsus (3 donkeys) and at the level of the fetlock joint (one donkey) and the medial aspect of the carpal joint (one donkey). According to the owners, all of these wounds were caused by falling down during walking and their age was about $3-5$ weeks.

Treatment procedures: Before treatment, wound area was calculated by multiplying the two largest dimensions to assess the initial size and evaluate the progress in the healing process (Table 1). Treatment of wounds was carried out in the following steps. (i) The whole area around the wound up to about $5 \mathrm{~cm}$ length from the wound edges was clipped and shaved. (ii) Grossly contaminated wounds were lavaged with body temperature tap water using a kitchen type spray nozzle over a grate to allow drainage.

Once the wound was lavaged, it was patted almost dry with a sterile towel. (iii) In the cases where an exuberant granulation tissue was found, a tourniquet was applied to the limb about $10 \mathrm{~cm}$ above the site of the wound and resection of the granulation or necrotic tissue was performed till the edges of the wound were higher than its center. Hemorrhage was stopped by pressure on the wound surface for 10 minutes. (iv) The wound surface was covered with a piece of gauze soaked in a mixture of an equal volume of cod liver oil (Western Pharmaceutical Industries, Egypt) and honey (pure raw untreated commercial clover honey obtained from Faculty of Agriculture, Assiut University, Egypt). The amount of the mixture varied according to the wound size. Generally, $20 \mathrm{ml}$ of the mixture was used for $100 \mathrm{~cm}^{2}$ dressing. (v) A protective pressure secondary bandage was applied to the wound surface including about $15 \mathrm{~cm}$ above and below the wound. (vi) The frequency of changing dressing was depended on how rapidly the mixture was diluted with exudate. In most cases, the bandage was changed periodically day after day for ten days, then every fourth day for two weeks and then once a week till the end of treatment and complete healing took place.

Treatment of surgically-induced wounds in donkeys with cod liver oil, honey or the mixture

To investigate the whole effect of the mixture of cod liver oil and honey on the healing of wounds, its effects were histologically compared with the effects of its two components, cod liver oil and honey. Eighteen surgically-induced wounds were made on the cranial aspects of the right fore metacarpus and the left hind metatarsus in 9 donkeys ( 2 - 3 years old). 

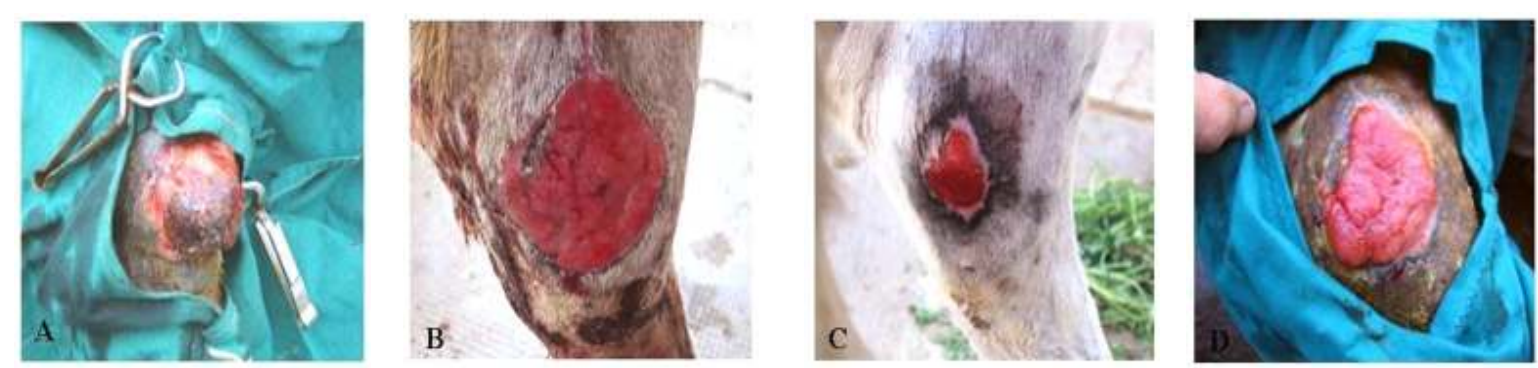

Figure. 1. Representative graphs for some clinically-treated wounds with mixture of cod liver oil and honey. A) Removal of massivelyformed exuberant granulation tissue. B) A clinically-treated wound showed no infection, cleanness and healthy surface and observable decrease in the wound surface after one week of treatment with the mixture. C) A clinically-treated wound appeared bright red in color moist and not elevated above the wound edges after 2 - 3 weeks of treatment. D) A clinically-treated wound showed raised center with granulation tissue that removed by second surgical debridement.

Animals were pre-medicated by intramuscular injection of chloropromazine $\mathrm{Hcl}$ (Neurazine, Miscv co., Egypt) in a dose of $0.4 \mathrm{mg} \mathrm{kg}$ b.w. followed by local infiltration analgesia of $10 \mathrm{ml}$ of $2 \%$ xylocaine at the site of skin incision. After aseptic preparation of the wound area, a $4 \mathrm{~cm}$ (radius) circular skin flap with subcutaneous tissue was removed. Hemorrhage was controlled by pressure on the wound surface for 10 minutes. Animals were divided into 3 groups (3 animals each). In each group, the three wounds at metacarpi were covered with pieces of gauze soaked in honey, cod liver oil and the mixture, respectively. The three parallel wounds at the metatarsi were left without treatment as untreated controls. Thereafter, wounds were similarly handled as in admitted animals. After healing completed, approximately after one month, wound area was calculated using ordinary ruler and hence animals were euthanized using chloral hydrate solution (15 g/kg b.w) and tissue specimens were taken by transecting the whole wound area and about $0.5 \mathrm{~cm}$ from the surrounding tissues. Tissue specimens were fixed in $10 \%$ neutral buffered formalin and then processed for histopathological examination. Briefly, tissue specimens were cut into longitudinal sections $(0.5 \mathrm{~cm}$ width), dehydrated in a graded alcohol series, cleared with methyl benzoate and embedded in paraffin wax. Five microns thick sections were cut and stained with hematoxylin and eosin (Bancroft and Stevens, 1990). Stained sections were examined under the light microscopy (Olympus CX31, Japan) and photographed using digital camera (Olympus, Camedia C-5060, Japan).

\section{Results}

Treatment of complicated wounds in admitted animals with mixture of cod liver oil and honey

In most treated cases with the mixture of cod liver oil and honey, healing of wounds took place in a period of time ranged from three to six weeks. It was found that surgical debridement at the beginning of treatment was important in most cases due to presence of massive exuberant granulation tissue (Fig. 1A). Application of tourniquets was found to be effective in controlling bleeding as the result of surgical debridement. However, some bleeding continued for a while after application of the first dressing bandage but it was minimal after the second dressing change to completely stop after one week of treatment. Lavage of the wound with body temperature tap water was seen to be very successful in removal of debris, necrotic tissue and pus as well as was helpful in refreshment of the wound surface.

After one week of treatment, wounds in 8 cases showed no infection, cleanness and healthy surface and observable decrease in the wound surface (Fig. 1B). After 2 - 3 weeks of treatment, the wound surface appeared bright red in color, moist and not elevated above wound edges (Fig. 1C). Wound areas were markedly decreased after one month of treatment (Table 1).

In two cases, newly-formed granulation tissue appeared raised above wound edges (Fig. 1D). Such granulation tissue was not as much as at the beginning of treatment and was removed by second surgical debridement.

Treatment of surgically-induced wounds with honey, cod liver oil or the mixture

Healing of surgically-induced wounds in all groups occurred in 3 - 4 weeks. In which, there were slight differences between different groups. Wounds treated with mixture of cod liver oil and honey showed some signs of inflammation such as swelling compared to the recently induced wound (Fig. 2A and B). Inflammation was little in wounds treated with honey and was unobservable in wounds treated with cod liver oil. Bleeding following the first dressing change was observed in all treated wounds (Fig. 2C). 

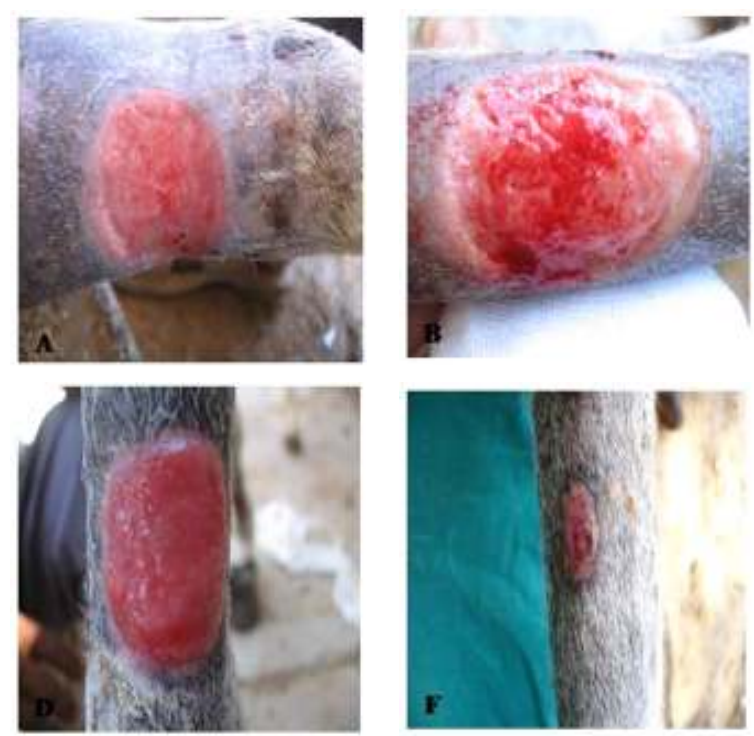

Slight increase of wound surface above the center of two wounds treated with the mixture was observed but returned to decrease after one week and did not need second surgical debridement (Fig. 2D and E). Healing process and decreasing of wound size were slightly faster in wounds treated with honey or cod liver oil compared to those treated with the mixture during the first two weeks and thereafter, no observable changes were seen. By the end of fourth week, significant decrease in the wound size was seen in all treated wounds (Table 2 and Fig. 2F). Irritation and signs of pain were seen in the wounds treated with honey alone during the first two weeks of treatment but not in those treated with cod liver oil or the mixture.

Histopathological examination of surgicallyinduced wounds treated with honey, cod liver oil or the mixture

Histopathological examination of tissue specimens was dealt with the two important processes in wound healing, epithelization and formation of granulation tissue. Using light microscopy, it was
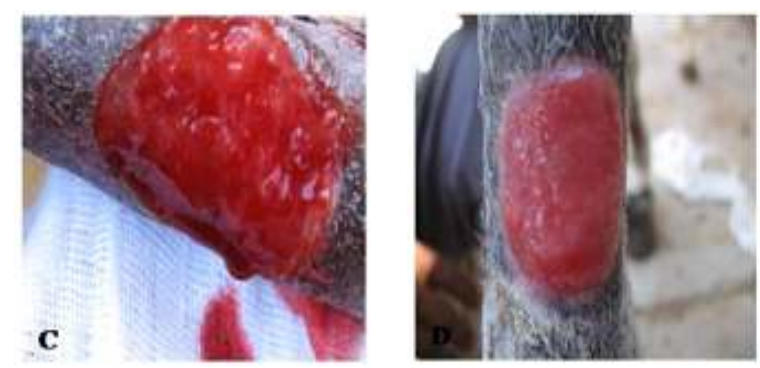

Figure.2. Representative graphs for some surgically-induced wounds treated with the mixture of cod liver oil and honey. A) A recent and untreated wound on metacarpal region of a donkey. B) A mixture-treated wound showed inflammatory swelling compared to the recently-induced wound. C) A mixture-treated wound showed bleeding after first dressing bandage. D) A mixturetreated wound showed slight increase in wound center with granulation tissue. E) A mixture treated-wound showed decrease in the size of granulation tissue without surgical debridement after one week. F) Significant decrease in the size of a mixture-treated wound by the end of third week.

found that all wounds treated with honey or cod liver oil showed complete epithelization over the wound areas after 4 weeks of wound induction (Fig. 3A and $\mathrm{B})$. On the other hand, wounds treated with mixture of cod liver oil and honey were not completely covered with epithelization and only epithelial cells extended from wound edges but did not cover the whole wound area (Fig. 3C). None of the control wounds at the metatarsi showed complete epithelization (Data not shown). The newly formed epithelium as the result of treatment with honey or cod liver oil consisted of 3 - 4 layers of cells. At this stage, these cells did not differentiate into distinct epidermal layers and appeared like spinous cells of fully differentiated epidermis with both round and flattened nuclei (Fig. 4A and B). In some situations, epithelial cells formed a thickened and hyperproliferative epithelium that consisted of two layers of cells. One layer appeared formed of tall cells located towards the basement membrane (likes stratum basal). The other group of
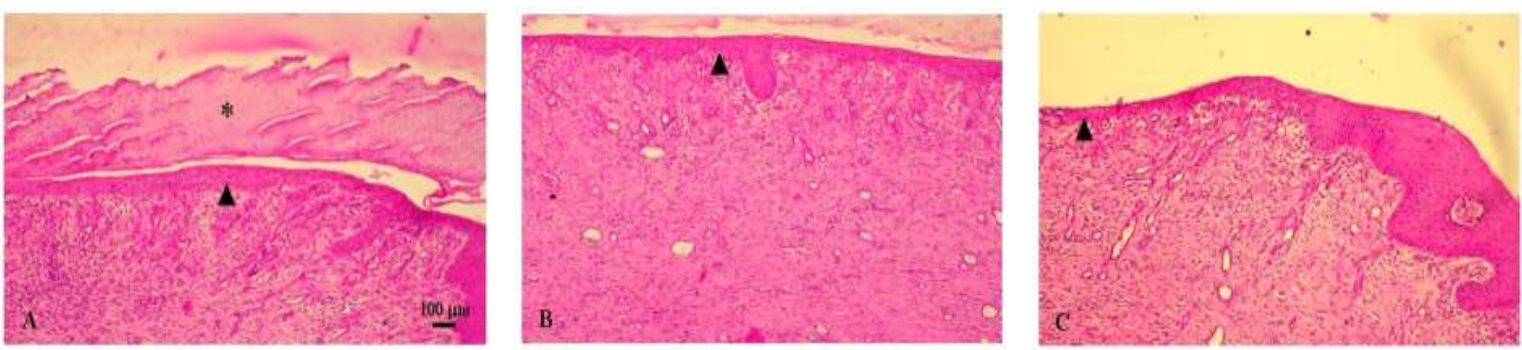

Figure-3. Representative micrographs for epithelization process as the result of treatment of surgically-induced wounds with honey, cod liver oil or their mixture. A) and B) Honey or cod liver oil induced complete epithelization (arrow head) over the wound areas. C) Mixture of cod liver oil and honey did not show complete epithelization (arrow head) and only epithelial cells extended from wound edges but did not cover the whole wound area. Note presence of a scab on the newly formed epithelium (asterisk). 

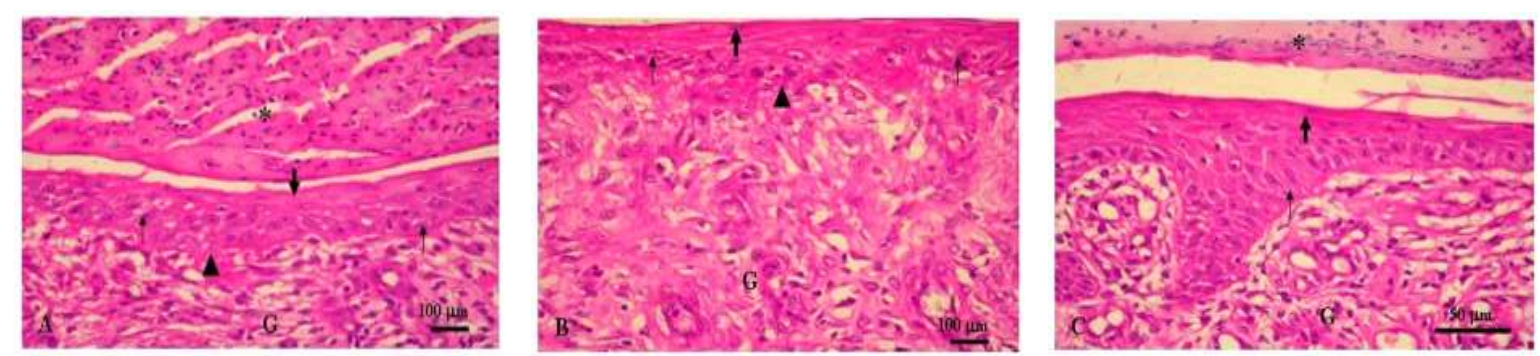

Figure-4. Representative micrographs for epithelization process as the result of treatment of surgically-induced wounds with honey, cod liver oil or their mixture. A) and B) Newly formed epithelium (arrow head) as the result of treatment of surgically-induced wounds with honey or cod liver oil appeared formed of 3 - 4 layers of spinous-like cells with either round (thin arrow) or flattened nuclei (thick arrow). C) A honey-treated wound showed a thickened and hyperproliferative epithelium that nearly differentiated into layers of stratum basal (thin arrow) and stratum spinosum (thick arrow) of epidermis. Note presence of a scab on the formed epithelium (asterisk) and underlying granulation tissue $(\mathrm{G})$.

cells were nearly spinous that appeared irregularly polygonal and separated by narrow translucent clefts which spanned by cytoplasmic spines (likes stratum spinosum) (Fig. 4C).

Histological examination of granulation tissue revealed some variability between different treatments. In honey-treated wounds, there were some evidences of inflammation, neovascularization and cross-linked fibroblasts (Fig. 5A). Granulation tissue in wounds treated with cod liver oil showed some degree of histological maturation consisted of newly formed blood capillaries, more fibroplasia and little fibroblasts (Fig. 5B). In mixture-treated wounds, granulation tissue in deep dermis consisted of neovas-cularization and variable numbers of fibroblasts and fibrocytes (Fig. 5C) while it showed higher degree of maturity in upper part with increasing numbers of fibrocytes and parallel collagenous fibers (Fig. 5D). In all examined wounds, there were no skin adenxa such as hair follicles and sweat and sebaceous glands after healing.

\section{Discussion}

Equines are known with their tendency to wounding probably due to their inquisitive nature,
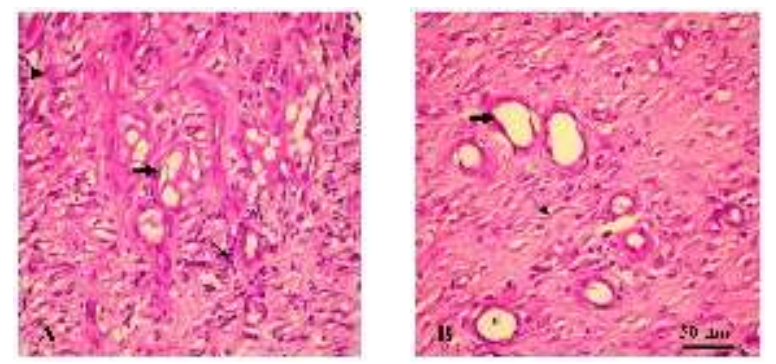

large size and confining in areas with potential obstacles such as metal or wire and their known difficulties with healing. The present study showed the beneficial effect of mixture of cod liver oil and honey in treatment of old traumatized wounds in equine and investigated the histological bases for such effect using standardized $4 \mathrm{~cm}$ (radius) full-thickness skin wounds in cranial aspects of both metacarpi and metatarsi. In clinically-treated wounds, it was found that surgical debridement and lavage were valuable steps in removing exuberant granulation tissue and debris and in minimizing infection. Formation of exuberant granulation tissue in wounds on lower limbs usually occurs as the result of weakness of the initial inflammatory response and the constriction of wound contraction, the two events that lead to chronic inflammation which further inhibits wound contraction and promotes exuberant granulation tissue formation (Wilmink and Weeren, 2005).

Application of mixture of cod liver oil and honey, after debridement, resulted in relieving edema and inflammation around the wound, remarkable decrease of exudation from the wound, disappearance of infection and observable decrease of wound surface
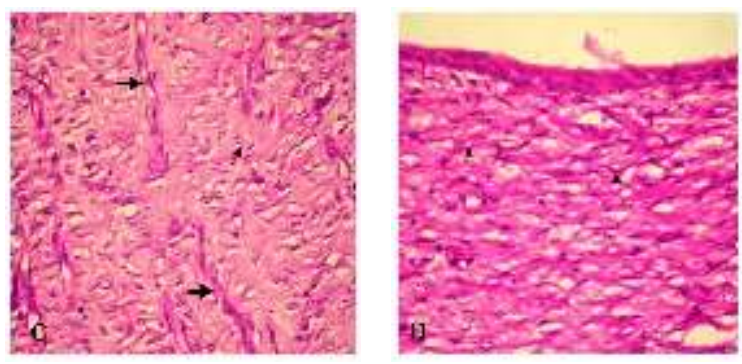

Figure-5. Representative micrographs for granulation tissue formation as the result of treatment of surgically-induced wounds with honey, cod liver oil or their mixture. A) A honey-treated wound showed some evidences of inflammation (thin arrow), neovascularization (thick arrow) and cross-linked fibroblasts (arrow head). B) Granulation tissue in a wound treated with cod liver oil showed some degree of histological maturation consisted of newly formed blood capillaries (thick arrow), more fibroplasias (thin arrow) and few fibroblasts. C) Deep dermis in a mixture-treated wound showed neovascularization (thick arrows) and variable numbers of fibroblasts and fibrocytes (thick arrow). D) A mixture-treated wound showed formation of highly matured granulation tissue with increasing numbers of fibrocytes and parallel collagenous fibers (thin arrow). 
after one week and significant reduction in wound size after 3 weeks of treatment in most cases. These effects of the mixture appeared to be mediated by the effects of honey, and vitamin A and omega-3 fatty acids in cod liver oil. In this context, Gethin et al. (2008) reported that honey dressings were associated with significant reduction in non-healed chronic superficial ulcers. Terkelsen et al. (2000) reported that vitamin A had an important role in accelerating wound healing. McDaniel et al. (2008) reported that omega-3 fatty acids in cod liver oil can affect wound healing through increasing pro-inflammatory cytokines production at wound sites. After application of the mixture, all wounds were covered with protective pressure secondary bandages which advantageous in controlling bleeding, reducing the tendency for exuberant granulation tissue formation, absorbing exudate, keeping the wound moist which helps epithelization, protecting the wound from contamination, dust and flies and keeping the topical mixture used better in contact with the wound surface. On the other hand, Dart et al. (2009) reported that bandaged wounds took longer time to heal than unbandaged wounds. This due to bandaging of wounds can result in greater and more prolonged retraction and excess granulation tissue formation (Dart et al., 2009) as well as can also compromise the immediate local and general blood supply (Knottenbelt, 1997).

In surgically-induced wounds, it was surprisingly found that honey or cod liver oil slightly accelerated healing process and decreased wound size compared to those treated with the mixture during the first two weeks to be equal at the end of third week. Initial acceleration in the healing process seen in wounds treated with honey or cod liver oil seemed to be attributed to the complete epithelization over the wound areas seen histologically, compared to partial epithelization occurred in the mixture-treated wounds. In parallel, Iftikhar et al. (2010) found that honey increased epithelization in wound models in rats. Moreover, Majtán et al. (2010) observed that honey increased metalloproteinase- 9 in cultured human keratinocytes. Metaloproteinase- 9 was observed to degrade type IV collagen in the basement membrane and further facilitate migration of keratinocytes (Kyriakides et al., 2009). Regarding cod liver oil, it was reported that topical application of cod liver oil ointment to surgically-induced full thickness dermal wounds on the ears of mice resulted in faster epithelization than those coated with Vaseline vehicle (Terkelsen et al., 2000). Vitamins A and D in cod liver oil seemed to be responsible of such effects. In consistency, Raju and Kulkarni (1998) and Ramesh et al. (1993) reported that vitamin A and cholecalciferol promoted epithelization in excision wounds in rats. As mentioned above mixture of cod liver oil and honey produced slower epithelization than honey or cod liver oil alone. However, the mixture produced good results in either clinically admitted or surgically induced wounds by the end of third week. This appeared due to formation of healthy scar that showed higher degree of maturity with an increasing number of fibrocytes and parallel collagen fibers.

In conclusion, cod liver oil/honey mixture was beneficial in healing of old traumatized wounds in equines. Adopting histopathological examination of surgically-induced wounds treated with honey, cod liver oil or their mixture revealed that the two former treatments accelerate epithelization and the mixture resulted in healthy scarring. Therefore, we recommend the mixture for treating old traumatized wounds as it enhances formation of healthy scar and cod liver oil or honey alone for recent surgical wounds as they promote epithelization.

\section{Acknowledgement}

The authors would like to thank the all staff as well as the assistant and workers of the Departments of Surgery and department of Histopathology, Faculty of Veterinary Medicine, Assiut, Egypt for their help and support.

\section{References}

1. Bancroft, J.D. and Stevans, A. (1990): Theory and Practice of HistologicalTechniques. $3^{\text {rd }}$ ed. pp. 113-305, Churchill Livingstone, Edinburgh.

2. Bigbie, R.B., Schumacher, J., Swaim, S.F., Purohit, R.C. and Wright, J.C. (1991): Effects of amnion and live yeast cell derivative on second-intention healing in horses. American Journal of Veterinary Research 52: 1376-1382.

3. Cavazana, W.C., Simões Mde, L., Amado, C.A. and Cuman, R.K. (2009): Sugar (sucrose) and fatty acid compounds with triglycerides on the treatment of wounds: experimental study in rats. Anals Brasileiros de Dermatologia 84: 229-236.

4. Cooper, R. and Molan, P. (1999): The use of honey as an antiseptic in managing Pseudomonas infection. Journal of Wound Care 8: 161-164.

5. Crowe, M.J., McNeill, R.B., Schlemm, D.J., Greenhalgh, D.G. and Keller, S.J. (1999): Topical application of yeast extract accelerates the wound healing of diabetic mice. The Journal of Burn Care and Rehabilitation 20: 155-162.

6. Dart, A.J., Perkins, N.R., Dart, C.M., Jeffcott, L.B. and Canfield, P. (2009): Effect of bandaging on second intention healing of wounds of the distal limb in horses. Australian Veterinary Journal 87: 215-218.

7. Knottenbelt, D.C. (1997): Equine wound management: are there significant differences in healing at different sites on the body? Veterinary Dermatology 8: 273-290.

8. Gethin, G.T., Cowman, S. and Conroy, R.M. (2008): The 
impact of Manuka honey dressings on the surface $\mathrm{pH}$ of chronic wounds. International Wound Journal 5: 185-194.

9. Gheldof, N. and Engeseth, N.J. (2002): Antioxidant capacity of honeys from various floral sources based on the determination of oxygen radical absorbance capacity and inhibition of in vitro lipoprotein oxidation in human serum samples. Journal of Agricultural and Food Chemistry 50: 3050-3055.

10. Iftikhar, F., Arshad, M., Rasheed, F., Amraiz, D., Anwar, P. and Gulfraz, M. (2010): Effects of acacia honey on wound healing in various rat models. Phytotherapy Research 24: 583-586.

11. Jacobs, K.A., Leach, D.H. and Fretz, P.B. (1984): Comparative aspects of the healing of excisional wounds on the leg and body of horses. Veterinary Surgery 13: 83-90.

12. Jorissen, M. and Bachert, C. (2009): Effect of corticosteroids on wound healing after endoscopic sinus surgery. Rhinology 47: 280-286.

13. Kietzmann, M. and Braun, M. (2006): Effects of the zinc oxide and cod liver oil containing ointment Zincojecol in an animal model of wound healing. Deutsche Tierärzliche Wochenschrift 113: 331-334.

14. Kyriakides, T.R., Wulsin, D., Skokos, E.A., Fleckman, P., Pirrone, A., Shipley, J.M., Senior, R.M. and Bornstein, P. (2009): Mice that lack matrix metalloproteinase-9 display delayed wound healing associated with delayed reepithelization and disordered collagen fibrillogenesis. Matrix Bioliology 28: 65-73.

15. Lay-flurrie, K. (2008): Honey in wound care: effects, clinical application and patient benefit. British Journal of Nursing 17: 32-36.

16. Liptak, J.M. (1997): An overview of the topical management of wounds. Australian Veterinary Journal 75: 408-413.

17. Majtán, J., Kumar, P., Majtán, T., Walls, A.F. and Klaudiny, J. (2010): Effect of honey and its major royal jelly protein 1 on cytokine and MMP-9 mRNA transcripts in human keratinocytes. Experimental Dermatology 19: $73-$ 79.

18. Majtán, J. (2009): Apitherapy--the role of honey in the chronic wound healing process. Epidemiologie Mikrobiologie Imunologie 58: 137-140.

19. McDaniel, J.C., Belury, M., Ahijevych, K. and Blakely, W. (2008): Omega-3 fatty acids effect on wound healing. Wound Repair Regeneration 16: 337-345.

20. Pieper, B. (2009): Honey-based dressings and wound care: an option for care in the United States. Journal of Wound Ostomy and Continence Nursing 36: 60-66.

21. Qunaibi, E.A., Disi, A.M. and Taha, M. O. (2009): Phenytoin enhances collagenization in excision wounds and tensile strength in incision wounds. Pharmazie 64: 584-586.

22. Raju, S.S. and Kulkarni, D.R. (1986): Vitamin A reverses the wound-healing suppressant effect of cyclophosphamide. Indian Journal of pharmacology 18: 154-157.

23. Ramesh, K.V., Mahindrakar, M.B. and Bhat, E.P. (1993): A new role for vitamin $\mathrm{D}$ : cholecalciferol promotes derma wound strength and re-epithelization. Indian Journal of Experimental Biology 31: 778-779.

24. Terkelsen, L.H., Eskild-Jensen, A., Kjeldsen, H., Barker, J.H. and Hjortdal, V.E. (2000): Topical application of cod liver oil ointment accelerates wound healing: an experimental study in wounds in the ears of hairless mice. Scandinavian Journal of Plastic and Reconstructive Surgury and Hand Surgurg 34: 15-20.

25. Wilmink, J.M. and van Weeren, P.R. (2005): Secondintention repair in the horse and pony and management of exuberant granulation tissue. The Veterinary Clinic of North America Equine Practice 21: 5-32.

26. Zumla, A. and Lulat, A. (1989): Honey - A remedy rediscovered. Journal of the Royal Society of Medicine 82: 384-385. 